\title{
Eyelid Carcinoma pN1b TNM Finding v8
}

National Cancer Institute

\section{Source}

National Cancer Institute. Eyelid Carcinoma pN1b TNM Finding v8. NCI Thesaurus. Code C140508.

Eyelid carcinoma with metastasis in a single ipsilateral regional lymph node, measuring 3 $\mathrm{cm}$ or less in greatest dimension based on lymph node biopsy. (from AJCC 8th Ed.) 